\title{
GENERAL POSITION THEOREMS FOR GENERALIZED MANIFOLDS
}

\author{
J. L. BRYANT
}

\begin{abstract}
It is an open question as to whether a generalized $n$-manifold, $n \geq 5$, that satisfies the disjoint disks property is a topological manifold. In this paper it is shown that any such space $X$ satisfies general position properties for maps of polyhedra into $X$.
\end{abstract}

The program to establish a topological characterization of euclidean space has been stalled, at least temporarily, by the discovery that Quinn's obstruction to resolving a generalized manifold may not be categorically zero as first asserted in [6]. (See [7].) Thus Cannon's conjecture [3] that a generalized $n$-manifold $X$, $n \geq 5$, is a topological manifold if and only if $X$ satisfies the disjoint disks property remains open. Quinn's results $[6,7]$, however, do produce an obstruction $\sigma(X)$ that vanishes if and only if $X$ is a topological manifold, although it is not known at this time whether the obstruction can be nonzero.

In this paper we show (Theorem 3 ) that any generalized $n$-manifold $X, n \geq 5$, having the disjoint disks property also satisfies general position properties for maps of arbitrary polyhedra into $X$. Thus, in a dimension theory sense $X$ behaves like a topological manifold. Theorems of this type have also been obtained by John Walsh [9].

DEFINITIONS. A generalized $n$-manifold, $n$-gm, is a euclidean neighborhood retract $X$ that is also a homology $n$-manifold; that is, $H_{*}(X, X-x) \cong$ $H_{*}\left(\mathbf{R}^{n}, \mathbf{R}^{n}-0\right)$ for all $x \in X$. (All homology is understood to have integer coefficients.) The space $X$ is said to satisfy the disjoint $k$-disks property, $\mathrm{DD}^{k} \mathrm{P}$ (or, simply, the disjoint disks property, DDP, when $k=2$ ) if for every pair of maps $f_{1}, f_{2}: D^{k} \rightarrow X$ of the unit $k$-disk $D^{k}$ of $\mathbf{R}^{k}$ into $X$ and $\varepsilon>0$, there are maps $f_{1}^{\prime}, f_{2}^{\prime}: D^{k} \rightarrow X$ such that $d\left(f_{i}^{\prime}, f_{i}\right)<\varepsilon$ and $f_{1}^{\prime}\left(D^{k}\right) \cap f_{2}^{\prime}\left(D^{k}\right)=\varnothing$. If $f, g: A \rightarrow X$ and $\varepsilon>0$, then $f \simeq_{\varepsilon} g$ means $f$ is $\varepsilon$-homotopic to $g$. A subset $A$ of $X$ is said to be 1-LCC in $X$ (1-locally coconnected in $X$ ) if for every $a \in A$ and neighborhood $U$ of $a$ in $X$ there is a neighborhood $V$ of $a$ in $X$ such that the inclusion induced homomorphism $\pi_{1}(V-A) \rightarrow \pi_{1}(U-A)$ is zero. A map (or embedding) $f: A \rightarrow X$ is said to be 1-LCC provided $f(A)$ is 1 -LCC in $X$.

Main results. In [3] Cannon shows that if $X$ is an $n$-gm, $n \geq 5$, having the DDP, then an arbitrary map of a 2-disk (hence, a 2-dimensional polyhedron) into

Received by the editors September 24, 1985.

1980 Mathematics Subject Classification (1985 Revision). Primary 57P05, 57N75; Secondary $57 Q 65$.

The author's research was partially funded by ONR grant \#N00014-84-K-0761. 
$X$ can be approximated by 1 -LCC embeddings. Our first result is

THEOREM 1. If $X$ is an $n$-gm, $n \geq 5$, satisfying the DDP, $P$ is a $k$-dimensional polyhedron, $2 k+1 \leq n$, and $f: P \rightarrow X$, then $f$ can be approximated by 1-LCC embeddings.

We show that an $n$-gm $X$ with the DDP is never "ghastly" [4].

THEOREM 2. Suppose $X$ is an $n$-gm, $n \geq 5$, having the $D D P, P$ is a $k$ dimensional polyhedron, and $f: P \rightarrow X$ is a map. Then $f$ can be approximated by a map $f^{\prime}: P \rightarrow X$ such that

(1) $\operatorname{dim} f^{\prime}(P) \leq k$, and

(2) $f^{\prime}(P)$ is $1-L C C$ if $k \leq n-3$.

Finally, we obtain the main general position property.

THEOREM 3. Suppose $X$ is an $n$-gm, $n \geq 5$, with the $D D P, P$ and $Q$ are polyhedra of dimensions $p$ and $q$, respectively, and $f: P \rightarrow X$ and $g: Q \rightarrow X$ are maps. Then $f$ and $g$ can be approximated by maps $f^{\prime}$ and $g^{\prime}$ such that

(1) $\operatorname{dim}\left[f^{\prime}(P) \cap g^{\prime}(Q)\right] \leq p+q-n$, and

(2) $f^{\prime}(P) \cap g^{\prime}(Q)$ is $1-L C C$ in $X$ if $p+q-n \leq n-3$.

Proofs of the main results. We start with a well-known fact (proved, for example, in $[2,8])$.

LEMMA 0. A locally compact, finite-dimensional, separable metric space $X$ admits a 1-LCC embedding in some euclidean space.

LEMMA 1. Suppose $X$ is an n-dimensional, 1-LCC closed subset of $\mathbf{R}^{m}, m-n \geq$ 3. Then there are $F_{\sigma}$ subsets $X_{0} \subset X_{1} \subset \cdots \subset X_{n}=X$ such that

(1) $\operatorname{dim} X_{i} \leq i$, and

(2) $\operatorname{dim}\left(X_{i}-X_{j}\right) \leq i-j-1$.

PROOF. Let $L_{k} \subset \mathbf{R}^{m}$ denote the Menger space:

$$
L_{k}=\left\{x \in \mathbf{R}^{m} \mid \text { at least } m-k \text { coordinates of } x \text { are rational }\right\} \text {. }
$$

Then $\operatorname{dim} L_{k}=k, L_{k}$ is a countable union of $k$-dimensional hyperplanes, and $\operatorname{dim}\left(\mathbf{R}^{m}-L_{k}\right)=m-k-1[5]$. Also $L_{0} \subset L_{1} \subset \cdots \subset L_{m}=\mathbf{R}^{m}$ and $\operatorname{dim}\left(L_{r}-L_{s}\right)=$ $r-s-1$. Since $X$ is $1-\mathrm{LCC}$ in $\mathbf{R}^{m}$, we can assume that $L_{k} \cap X=\varnothing$ for $k \leq m-n-1$ $[2,8]$.

Set $X_{i}=X \cap L_{m-n+i}$. Then

$$
\begin{aligned}
\operatorname{dim} X_{i} & =\operatorname{dim}\left(X \cap L_{m-n+i}\right) \\
& =\operatorname{dim}\left(X \cap\left(L_{m-n+i}-L_{m-n-1}\right)\right) \\
& \leq(m-n+i)-(m-n-1)-1=i,
\end{aligned}
$$

and

$$
\begin{aligned}
\operatorname{dim}\left(X_{i}-X_{j}\right) & =\operatorname{dim}\left(X \cap\left(L_{m-n+i}-L_{m-n+j}\right)\right) \\
& \leq \operatorname{dim}\left(L_{m-n+i}-L_{m-n+j}\right)=i-j-1 .
\end{aligned}
$$

Throughout the rest of this paper $X$ is an n-gm satisfying the DDP, 1-LCC embedded in $\mathbf{R}^{m}$ for some $m \geq n+3$. 
LEMMA 2. There are $F_{\sigma}$ subsets $X_{0} \subset X_{1} \subset \cdots \subset X_{n}=X$ such that

(1) $\operatorname{dim} X_{i} \leq i$

(2) $\operatorname{dim}\left(X_{i}-X_{j}\right) \leq i-j-1$, and

(3) $X_{i}$ is 1-LCC in $X$ for $i \leq n-3$.

PROOF. Let $\left\{f_{i}\right\}_{i=1}^{\infty}$ be a countable dense subset of $\operatorname{Map}\left(D^{2}, X\right)$, the space of maps (with the compact-open topology), consisting of 1-LCC embeddings [3]. In the proof of Lemma 1 , require that $L_{k} \cap f_{i}\left(D^{2}\right)=\varnothing$ for $k \leq m-3$ and all $i$ (using the fact that the 1-LCC property is transitive). Then $X_{i} \cap f_{j}\left(D^{2}\right)=\varnothing$ for $i \leq n-3$. Thus $X_{i}$ is 1-LCC in $X$ for $i \leq n-3$.

LEMMA 3. Let $X_{0} \subset X_{1} \subset \cdots \subset X_{n}=X$ be $F_{\sigma}$ subsets of $X$ satisfying the conclusion of Lemma 2. Then $\pi_{k}\left(X, X-X_{i}\right)=0$ for $k<n-i$.

Proof. By local duality in $X[\mathbf{1}, \mathbf{1 0}], H_{k}\left(X, X-X_{i}\right)=0$ for $k<n-i$. If $i=n-1$ or $n-2$, then $\pi_{k}\left(X, X-X_{i}\right)=H_{k}\left(X, X-X_{i}\right)=0$. If $i \leq n-3$, then $X_{i}$ is 1-LCC in $X$ so that $\pi_{2}\left(X, X-X_{i}\right)=0$. Thus by the Hurewicz isomorphism theorem, $\pi_{k}\left(X, X-X_{i}\right)=0$ for $i \leq n-3$ and $k<n-i$. (We may assume that $X$, and hence $X-X_{i}, i \leq n-3$, are simply connected by passing to the universal cover of $X$.)

LeMma 4. Suppose $f: D^{k} \rightarrow X$ is a map and $\varepsilon>0$. Then $f \simeq_{\varepsilon} f^{\prime}$ such that $f^{\prime}\left(D^{k}\right) \cap X_{n-k-1}=\varnothing$ and $f^{\prime}$ is 1-LCC, if $k \leq n-3$.

ProOF. To get $f^{\prime}\left(D^{k}\right) \cap X_{n-k-1}=\varnothing$, apply Lemma 3 locally, using induction on the skeleta of a fine triangulation of $D^{k}$. Similarly, if $D$ is a 1-LCC 2-cell in $X$, then for a relative neighborhood $U$ of $\operatorname{Int} D,(\operatorname{rel} \operatorname{Bd} D), \pi_{2}(U, U-\operatorname{Int} D)=0$ and $H_{i}(U, U-\operatorname{Int} D)=0$ for $i \leq n-3$. Thus for $r \leq n-3$ any map $g:\left(D^{r}, \operatorname{Bd} D^{r}\right) \rightarrow$ $(U, U-\operatorname{Int} D)$ is homotopic $\operatorname{rel~Bd} D^{r}$ to a map $g^{\prime}: D^{r} \rightarrow U-\operatorname{Int} D$. Applying this argument inductively on the skeleta of a fine triangulation of $D^{k}$, we can get $f \simeq_{\varepsilon} f^{\prime}$ such that $f^{\prime}\left(D^{k}\right) \cap D=\varnothing$ for any 1-LCC 2-cell $D$ in $X$. Thus we may simultaneously obtain $f \simeq_{\varepsilon} f^{\prime}$ so that $f^{\prime}$ is 1-LCC and $f^{\prime}\left(D^{k}\right) \cap X_{n-k-1}=\varnothing$.

COROLlaRY. If $f: D^{k} \rightarrow X$ and $\varepsilon>0$, then $f \simeq \varepsilon f^{\prime}$ where $f^{\prime}$ is $1-L C C$, if $k \leq n-3$, and $\operatorname{dim} f^{\prime}\left(D^{k}\right) \leq k$.

The proof of Theorem 2 follows immediately.

LeMma 5. $X$ satisfies $D D^{k} P$ for $k<n / 2$.

ProOF. Given $f, g: D^{k} \rightarrow X$, apply the Corollary to get $f \simeq_{\varepsilon} f^{\prime}$ where $f^{\prime}$ is 1-LCC and $\operatorname{dim} f^{\prime}\left(D^{k}\right) \leq k$. Then for any open set $U$ of $X, \pi_{i}\left(U, U-f^{\prime}\left(D^{k}\right)\right)=0$ for $i \leq n-k-1$. If $k<n / 2$, then $k \leq n-k-1$ and, hence, by a now familiar argument, for any $\varepsilon>0, g \simeq_{\varepsilon} g^{\prime}$ where $g^{\prime}\left(D^{k}\right) \cap f^{\prime}\left(D^{k}\right)=\varnothing$.

The proof of Theorem 1 now follows easily as in [3].

PROOF OF THEOREM 3 . It is clearly sufficient to prove the theorem when $P$ is a $p$-simplex and $Q$ is a $q$-simplex. Suppose $f: P \rightarrow X$ and $g: Q \rightarrow X$ are maps. Let $X_{0} \subset X_{1} \subset \cdots \subset X_{n}=X$ be $F_{\sigma}$ subsets of $X$ obtained from Lemma 2. Then $f$ can be approximated by $f^{\prime}: P \rightarrow X$ such that $f^{\prime}(P) \cap X_{i} \subset X_{i}-X_{n-p-1}$, by applying Lemma 3.

Observe that $f^{\prime}(P) \cap X_{2 n-p-q-1}$ is a $\sigma$-compact subset of $X_{2 n-p-q-1}-X_{n-p-1}$, which has dimension $n-q-1$. We wish to approximate $g$ by $g^{\prime}: Q \rightarrow X$ so that $g^{\prime}(Q) \cap f^{\prime}(P) \cap X_{2 n-p-q-1}=\varnothing$, for then it would follow that $g^{\prime}(Q) \cap f^{\prime}(P) \subset$ $X-X_{2 n-p-q-1}$, which has dimension $p+q-n$, as desired. 
Recall that $L_{r}=\left\{x \in \mathbf{R}^{m} \mid\right.$ at most $m-r$ coordinates of $x$ are rational $\}=$ $\bigcup_{j=1}^{\infty} E_{j}$, where $E_{1} \subset E_{2} \subset \cdots$ and each $E_{j}$ is a finite union of $r$-dimensional hyperplanes [5]. Thus each $X_{i}=\bigcup_{j=1}^{\infty} Y_{i j}$ where $Y_{i j}$ is a closed subset of $X$ of dimension $\leq i$ and $Y_{i j}$ is 1-LCC in $X$ if $i \leq n-3$. For $i=2 n-p-q-1, Y_{i j} \cap f^{\prime}(P)$ is a compact set of dimension $\leq n-q-1$.

Assume first that $i=2 n-p-q-1 \leq n-3$. Then $H_{k}\left(X, X-\left(Y_{i j} \cap f^{\prime}(P)\right)\right)=0$ for $0 \leq k \leq q$ and so $\pi_{k}\left(X, X-\left(Y_{i j} \cap f^{\prime}(P)\right)\right)=0$ for $0 \leq k \leq q$; hence, we can choose $g^{\prime}: Q \rightarrow X$ approximating $g$ so that $g^{\prime}(Q) \cap\left(Y_{i j} \cap f^{\prime}(P)\right)=\varnothing$ for all $j$. That is, $g^{\prime}(Q) \cap f^{\prime}(P) \cap X_{2 n-p-q-1}=\varnothing$.

Suppose next that $i=2 n-p-q-1=n-2, p \leq q$ (without loss of generality), and $n \geq 6$. Then $n-p \geq 3$ and we can assume further that $f^{\prime}(P)$ and, hence, $f^{\prime}(P) \cap Y_{i j}$ are 1-LCC in $X$. From this we see that $\pi_{k}\left(X, X-\left(f^{\prime}(P) \cap Y_{i j}\right)\right)=0$ for $0 \leq k \leq q$ and so we can find $g^{\prime}$ such that $g^{\prime}(Q) \cap f^{\prime}(P) \cap Y_{i j}=\varnothing$.

Suppose $2 n-p-q-1=n-1, n \geq 5$, and $p \leq q$. Then again $n-p \geq 3$ and the above argument works.

Finally, if $2 n-p-q-1=n-2$ and $n=5$, then the only case in which the above argument does not work is $p=q=3$.

Suppose then that $n=5$ and $p=q=3$. Let $\left\{f_{i}\right\}_{i=1}^{\infty}$ be a countable dense subset of $\operatorname{Map}\left(D^{2}, X\right)$ consisting of 1-LCC embeddings. Given $f: P \rightarrow X$ and $g: Q \rightarrow X$, approximate $f$ by $f^{\prime}$ such that $\operatorname{dim}\left(f^{\prime}(P) \cap f_{i}\left(D^{2}\right)\right) \leq 0$ for all $i=1,2, \ldots$ and $\operatorname{dim} f^{\prime}(P) \leq 3$. Then $f^{\prime}(P)$ and $f^{\prime}(P) \cap f_{i}\left(D^{2}\right), i=1,2, \ldots$, are 1-LCC in $\mathbf{R}^{m}$ since $X$ is. Thus for any $\varepsilon>0$ it is possible to find an $\varepsilon$-homeomorphism $h: \mathbf{R}^{m} \rightarrow \mathbf{R}^{m}$ such that $h\left(f^{\prime}(P) \cap f_{i}\left(D^{2}\right)\right) \cap L_{m-2}=\varnothing, i=1,2, \ldots, h(X) \cap L_{k}=\varnothing$, for $k \leq m-4$, and $h\left(f^{\prime}(P)\right) \cap L_{m-2}$ is a 1-dimensional $\sigma$-compact set that is 1-LCC in $X$.

Now replace $X_{i}=X \cap L_{m-5+i}$ by $Y_{i}=X \cap h^{-1}\left(L_{m-5+i}\right)$. Then $f^{\prime}(P) \cap Y_{3}$ is a 1-dimensional $\sigma$-compact set that is 1-LCC in $X$. Hence, $g$ can be approximated by $g^{\prime}: Q \rightarrow X$ such that $g^{\prime}(Q) \cap f^{\prime}(P) \cap Y_{3}=\varnothing$. This means that $g^{\prime}(Q) \cap f^{\prime}(P) \subset$ $X-Y_{3}$, which has dimension 1 .

\section{REFERENCES}

1. G. E. Bredon, Sheaf theory, McGraw-Hill, New York, 1967.

2. J. Bryant, On embeddings of compacta in euclidean space, Proc. Amer. Math. Soc. 23 (1969), 46-51.

3. J. W. Cannon, Shrinking cell-like decompositions of manifolds. Codimension three, Ann. of Math. (2) 110 (1979), 83-112.

4. R. J. Daverman and J. Walsh, A ghastly generalized $n$-manifold, Illinois J. Math. 25 (1981), $555-576$.

5. W. Hurewicz and H. Wallman, Dimension theory, Princeton Univ. Press, Princeton, N. J., 1948.

6. F. Quinn, Resolutions of homology manifolds and the topological characterization of manifolds, Invent. Math. 72 (1983), 267-284.

7. __ An obstruction to the resolutions of homology manifolds, preprint.

8. M. A. Stan'ko, The embedding of compacta in euclidean space, Mat. Sb. 83 (125) (1970), 234-255 (=Math. USSR-Sb. 12 (1970), 234-255).

9. J. Walsh, General position properties of generalized manifolds: a primer, Proc. Idaho State Topology Conf., 1985.

10. R. L. Wilder, Topology of manifolds, Amer. Math. Soc. Colloq. Publ., vol. 32, Amer. Math. Soc., Providence, R.I., 1949.

Department of Mathematics, Florida State University, Tallahassee, FlorIDA 32306 